\title{
Context and competition in the capture of visual attention
}

\author{
Clayton Hickey • Jan Theeuwes
}

Published online: 8 July 2011

(C) The Author(s) 2011. This article is published with open access at Springerlink.com

\begin{abstract}
Competition-based models of visual attention propose that perceptual ambiguity is resolved through inhibition, which is stronger when objects share a greater number of neural receptive fields (RFs). According to this theory, the misallocation of attention to a salient distractorthat is, the capture of attention - can be indexed in RF-scaled interference costs. We used this pattern to investigate distractor-related costs in visual search across several manipulations of temporal context. Distractor costs are generally larger under circumstances in which the distractor can be defined by features that have recently characterised the target, suggesting that capture occurs in these trials. However, our results show that search for a target in the presence of a salient distractor also produces RF-scaled costs when the features defining the target and distractor do not vary from trial to trial. Contextual differences in distractor costs appear to reflect something other than capture, perhaps a qualitative difference in the type of attentional mechanism deployed to the distractor.
\end{abstract}

Keywords Attention · Visual search · Capture · Biased competition

Our visual environment is rich with interesting objects, but our visual system is fundamentally limited in its ability to concurrently process information stemming from multiple objects at the same time (Broadbent, 1958; Treisman, 1969). This limitation results in part from the receptive field (RF) organisation of visual cortex. Visual processing is

\footnotetext{
C. Hickey $(\bowtie) \cdot J$. Theeuwes

Department of Cognitive Psychology,

Vrije Universiteit Amsterdam,

Van der Boechorststraat 1,

1081 HV Amsterdam, The Netherlands

e-mail: c.hickey@psy.vu.nl
}

organised hierarchically, such that neurons in high-level visual areas consolidate information from neurons in lowlevel areas. As the information encoded by any single neuron becomes more complex, the RF associated with that neuron becomes correspondingly larger in size (Desimone \& Ungerleider, 1989). Eventually this creates a problem: When stimuli are close enough that they fall within the same RF, and therefore are represented by the same cell, neural coding becomes ambiguous (Desimone \& Duncan, 1995; Luck, Girelli, McDermott, \& Ford, 1997). Output from that cell can no longer be attributed to a single object but may stem from any of the stimuli that fall within its RF.

Processing limitations in visual cortex create the need for a selective mechanism-visual attention-responsible for limiting input into late stages of visual processing and thus resolving neural ambiguity. Several influential models of visual attention, including the biased-competition framework of Desimone and Duncan (1995), propose that attention is instantiated in the competition of object representations (see also Luck et al., 1997; Tsotsos et al., 1995). According to this idea, raw salience drives neurons responsible for representing visual objects to inhibit one another, with the result that high-salience items are more likely to be represented in late processing than are lowsalience items. Endogenous biases of this competitive process ensure that low-salience objects with behavioural relevance will continue to be represented in the system.

Single-unit and neuroimaging studies have provided substantial support for the idea of endogenous biases in competition. In monkey electrophysiology, biases are evident in the reduction of a neuron's response to an effective stimulus - a stimulus that the neuron normally responds to - when attention is paid to another, noneffective stimulus presented in the neuron's RF (Chelazzi, Miller, Duncan, \& Desimone, 1993; Luck et al., 1997; Miller, 
Gochin, \& Gross, 1993; Moran \& Desimone, 1985; Reynolds, Chelazzi, \& Desimone, 1999). Evidence from neuroimaging work with humans relies on different logic; here, inhibition is often indexed as a reduced hemodynamic response in an area of visual cortex (such as a portion of V4) when stimuli are concurrently rather than sequentially presented. Endogenous biases become apparent when attention is paid to one of several concurrently presented stimuli. Under these circumstances, inhibition is no longer evident, suggesting that the deployment of attention to a target reduces the inhibitory influence of surrounding stimuli (Kastner, De Weerd, Desimone, \& Ungerleider, 1998; Kastner et al., 2001).

The effect of salience on competition is much the same as the effect of the endogenous deployment of attention. This was demonstrated in monkey cortex by Reynolds and Desimone (2003), who had monkeys view two stimuli that fell within the same V4 receptive field. When the stimuli were unattended, neural response in V4 cells was dominated by the higher-contrast of the two stimuli. Critically, when the two stimuli were of equal contrast and attention was deployed to one of them, the cellular response was driven by the attended object in much the same way as it had been by the high-contrast stimulus. The effect of attention was equivalent to a $50 \%-79 \%$ increase in stimulus contrast. Beck and Kastner (2005) demonstrated the same principle in humans using functional neuroimaging. Here the presence of a uniquely coloured item presented among homogeneous distractors - a type of object known in the literature as a colour singleton-was found to reduce mutual inhibition in much the same way as occurs when attention is deployed (Kastner et al., 1998). The colour singleton had a larger impact on inhibition than did the deployment of attention, in line with the idea that salience plays a critical role in the resolution of visual information.

The importance of salience in neural competition is consistent with the body of literature suggesting that salient, task-irrelevant visual objects can capture attention to their location during visual search (e.g., Hickey, McDonald, \& Theeuwes, 2006; Theeuwes, 1991, 1992; for recent reviews, see Burnham, 2007; Rauschenberger, 2003; Theeuwes, 2010). The idea here is that salience might drive interactions early in time, such that the distractor is better represented in the system than the target. This would cause the need for a subsequent endogenous rebiasing of competitive interactions in order that target discrimination could occur.

Theeuwes (1991) provided early results in line with this proposal. Participants in this study were presented with displays that contained a shape singleton target-a uniquely shaped object-among a number of low-salience distractors. Often the target was the only singleton in the display, but in many trials one of the distractor stimuli was given a unique colour, in this way defining a salient, task-irrelevant colour singleton. Results showed that responses to the target were slower and more error prone when the salient distractor was present, consistent with the idea that attention was initially misallocated to the distractor. This paradigm has been used in a number of subsequent studies (e.g., Bacon \& Egeth, 1994; de Fockert, Rees, Frith, \& Lavie, 2004; Hickey, Chelazzi, \& Theeuwes, 2010; Hickey et al., 2006) and has become known in the literature as the additional-singleton paradigm.

Theeuwes $(1991,1992,2004)$ has consistently interpreted distractor costs in the additional-singleton paradigm as evidence of the capture of spatial attention to the location of the salient distractor, but other interpretations have been offered. One of the most compelling of these was suggested by Folk and Remington (1998), who proposed that the costs were due to a perceptual filtering process similar to that reported in Kahneman, Treisman, and Burkell (1983). According to this idea, the increased perceptual complexity of visual search displays containing a salient distractor causes a need for perceptual resolution that completes prior to the deployment of attention. Critically, according to this idea, the salient distractor does not capture attention.

On the surface, the perceptual-filtering hypothesis might be framed within the context of biased competition-if, for example, perceptual filtering occurred through competitive interaction between stimuli (though this has not been made explicit in the literature). However, there are some problems with this type of reconciliation. For example, biased competition is a distinctly spatial operation, with RFs effectively acting as resource units. The spatial nature of the perceptualfiltering account suggested by Folk and Remington (1998) is not well defined. More importantly, biased competition proposes that the resolution of neural ambiguity through competitive interaction is visual attention. In contrast, the perceptual-filtering hypothesis proposes that filtering occurs prior to the deployment of attention.

An opportunity for integration might be provided by behavioural studies of target-distractor competition. A pervasive finding in this work has been that RT suffers when a visual search target comes to be nearer to a salient distractor (Caputo \& Guerra, 1998; McCarley \& Mounts, 2007, 2008; Mounts, 2000a, 2000b, 2005; Mounts \& Gavett, 2004). This proximity effect is attributed to the misallocation of attention: Objects close to one another share a greater number of RFs than do objects far from one another, and this causes increased ambiguity. When attention is misallocated to the distractor, this results in inhibition of the target that is scaled according to the number of shared RFs. This inhibition slows the corrective redeployment of attention to the target, and when this inhibition grows stronger, the effect is exacerbated. Mounts (2000a) provided a clear example of this process. In this 
visual search study, the target was presented alongside a salient distractor. When the target was a singleton and the distractor was a more salient singleton, proximity effects were observed. Under these circumstances, participants likely adopted a search strategy based on salience, such that the distractor captured attention (singleton detection mode; Bacon \& Egeth, 1994). In contrast, when the target was defined by a conjunction of features, and a saliencebased search strategy was therefore not suitable, the presence of the singleton distractor had a static effect on $\mathrm{RT}$, and proximity effects were no longer apparent.

The static distractor cost observed in Mounts (2000a) and similar work might reflect something akin to the perceptual filtering proposed by Folk and Remington (1998). According to this idea, the presence of a salient distractor might slow the initial deployment of attention, which would result in behavioural distractor costs even in the absence of attentional capture. This possibility is consistent with results from analysis of temporal context in the additional-singleton paradigm. Distractor costs are large in mixed blocks of the task, when the visual features used to define the target on any one trial can be used to define a distractor in subsequent trials (Hickey et al., 2006; Theeuwes, 1991). These costs are substantially reduced in pure blocks, where the target and distractor are consistently defined by unique features (Theeuwes, 1992). This pattern has been linked to intertrial priming (Hillstrom, 2000; Maljkovic \& Nakayama, 1994), with much of the difference between mixed and pure blocks stemming from "swap" trials, where the features that define the distractor characterised the target in the immediately preceding trial (and vice versa; Hickey, Olivers, Meeter, \& Theeuwes, 2011; Pinto, Olivers, \& Theeuwes, 2005). However, the idea that attention is captured only in "swap" trials does not explain the residual distractor costs observed in "same" trials. These may reflect a perceptual cost unrelated to attentional capture.

The present study was designed to investigate the sources of distractor costs in competitive visual search. Based on the existing literature (e.g., Caputo \& Guerra, 1998; McCarley \& Mounts, 2007, 2008; McCarley, Mounts, \& Kramer, 2007; Mounts, 2000a, 2000b, 2005; Mounts \& Gavett, 2004), we approached experimentation with the idea that capture can be indexed in RF-scaled effects, and thus that the presence or absence of this pattern might allow us to distinguish between circumstances in which distractor costs are caused by capture and circumstances in which distractor costs stem from some other source.

We measured RF-scaled effects in two ways, first by analysing RT as a function of target-distractor proximity, and second by analysing RT as a function of whether target and distractor were presented to the same visual hemifield or to contralateral visual hemifields. The hemispheric organisation of the brain is such that few visual RFs cross the vertical meridian of the visual field (Desimone \& Ungerleider, 1989), and as a result, stimuli presented to one visual hemifield share a greater number of RFs on average than do stimuli presented to contralateral hemifields (Mounts \& Gavett, 2004; Torralbo \& Beck, 2008). Hemispheric effects therefore provide a measure of RFscaled, capture-induced interference in addition to proximity effects.

In Experiment 1, we had participants take part in a mixed-block version of the additional-singleton paradigm in which the target and distractor colours could swap between trials. The analysis included a partitioning of trials into a "swap" condition, where the colour that defined the distractor had defined the target in the previous trial (and vice versa), and a "same" condition, in which the colours that defined the target and distractor were the same as those employed in the previous trial. Based on the idea that capture occurs when the distractor is defined by features that have characterised the target (Hickey et al., 2011; Pinto et al., 2005), our expectation was that RF-scaled effects would be evident in "swap" trials and absent in "same" trials.

\section{Experiment 1}

\section{Method}

Participants A group of 18 neurologically normal volunteers were financially compensated for their participation. Four of the participants were excluded from analysis, 2 because of excessively long reaction times $(>2,500 \mathrm{~ms}$ median RT) and 2 because of an apparent inability to complete the task while maintaining eye fixation. All of the remaining 14 participants $(6$ women, 8 men; mean age = 21 years $\pm 3.2 S D$ ) were right-handed. ${ }^{1}$

Stimuli and procedure The stimuli and task instructions were identical to those in Theeuwes (1991). The primary experimental display was a visual search array consisting of 10 discrete shape stimuli, each presented equidistant from a central fixation point $\left(9.1^{\circ}\right)$ and from one another $\left(5.6^{\circ}\right.$; see Fig. 1). The shape stimuli were unfilled diamonds $\left(4.2^{\circ} \times\right.$ $\left.4.2^{\circ}\right)$ and circles $\left(1.7^{\circ}\right.$ radius) with thin $\left(0.3^{\circ}\right)$ red or green outlines. A grey line $\left(0.3^{\circ} \times 1.5^{\circ}\right)$ randomly oriented either vertically or horizontally was contained within each of the shape stimuli. Participants searched for a uniquely shaped stimulus presented among eight or nine identical distrac-

\footnotetext{
${ }^{1}$ Results from analysis of the electroencephalographic data recorded while participants completed Experiment 1 have been reported in Hickey et al. (2006, Exp. 1).
} 
Fig. 1 Example of the stimulus array for a distractor-present trial. A unique colour is denoted by the broken line

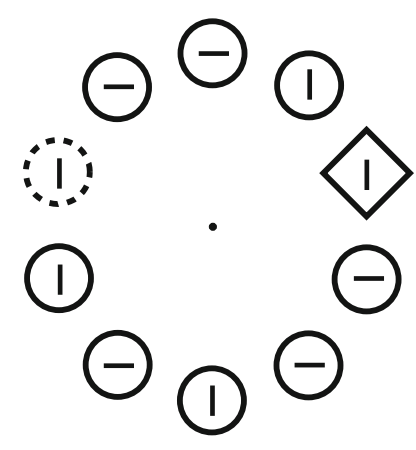

tors. In each trial, the target was equally likely to be a circle among diamonds or a diamond among circles, and a target was present in every trial. In $66 \%$ of the trials, a uniquely coloured distractor singleton was also present in the display. The distractor singleton was of the same shape as all of the other distractors but was either red, with all other stimuli green, or vice versa. Distractor-present and distractor-absent trials were randomised within blocks.

The experimental stimuli were presented on a CRT monitor located $60 \mathrm{~cm}$ from the observer's eyes. Each experimental trial began with the presentation of a fixation point for a random duration of $600-1,600 \mathrm{~ms}$, which was followed by presentation of a visual search array. The visual search array remained on the screen until $100 \mathrm{~ms}$ after a response was made, at which point the next trial began. Participant response was based on the orientation of the line contained within the shape singleton. Because line orientation was randomised, the target contained a vertical line in approximately half of trials and a horizontal line in the remainder. Participants were instructed to respond as

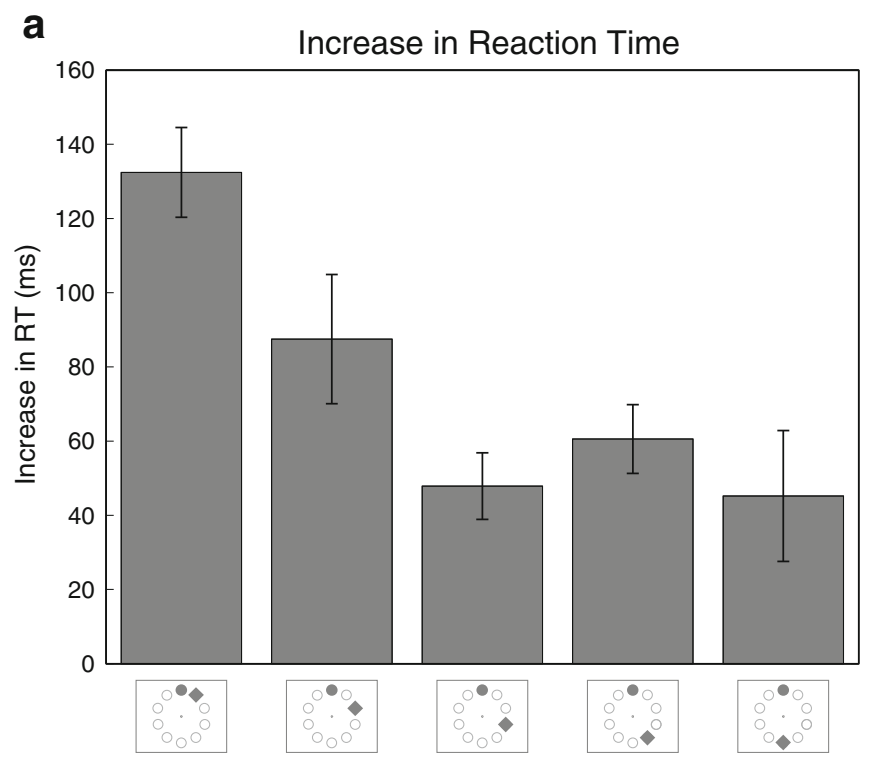

Fig. 2 Results from the proximity analysis for Experiment 1, showing increases in both (a) reaction times (RTs) and (b) error rates. Here, as in all figures for Experiment 1, increases in RTs or error rates are quickly as possible while maintaining an average accuracy of $90 \%$ or better, and feedback regarding accuracy and response latency was given at the end of each experimental block. Each experimental block consisted of 48 trials, and each participant completed 30 experimental blocks, for a total of 1,440 experimental trials per participant. Prior to beginning the experiment, each participant completed at least one practice block. All participants responded with their right hand by pressing the left mouse button when the target line was vertical and the right mouse button when the line was horizontal. Eye fixation was required of participants during the experiment and was monitored via electrooculogram (EOG); trials containing vertical or horizontal eye movements were rejected from the analysis, as were trials in which response latencies exceeded $1.35 \mathrm{~s}$.

\section{Results}

Participants were slower and more error prone when visual search arrays contained a distractor singleton [mean median RT: 789 vs. $872 \mathrm{~ms}, t(13)=9.14, p<.001$; error: $7.1 \%$ vs. $10.8 \%, t(13)=4.83, p<.001]$. In order to examine proximity effects in the data, we binned results as a function of target-distractor distance. Proximity ranged over five levels, from trials on which the target and distractor were presented side by side to trials on which the target and distractor were opposite in the display (see the bottom of Fig. 2 for examples). Median RTs and error rates were computed for the five proximity conditions and compared to behavioural measures observed in the

b

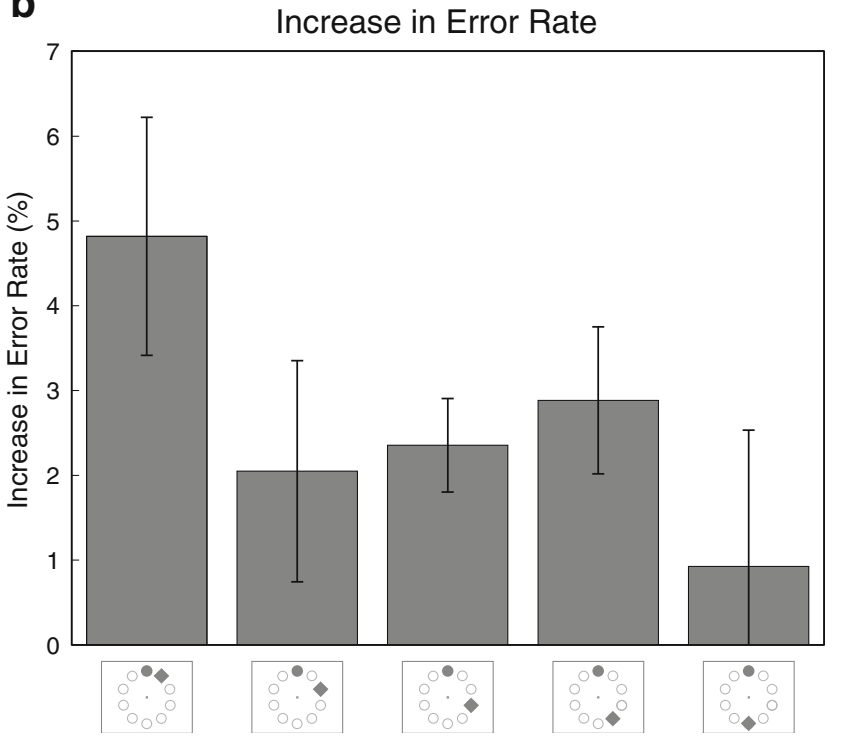

relative to the distractor-absent condition. As in all figures for this study, error bars reflect standard errors of the means 
distractor-absent condition. This procedure yielded the difference scores illustrated in Fig. 2. RTs and errors increased as the distance between target and distractor decreased. This pattern was significant in the statistical analysis; repeated measures analyses of variance (RANOVAs) revealed a main effect of proximity for $\operatorname{RT}[F(4,52)=$ $9.35, p<.001]$ and a marginally significant effect of proximity for error rate $[F(4,52)=2.37, p=.064]$.

We further analysed the proximity results as a function of intertrial context. Because prior work had suggested that change in colour underlies much of the intertrial effect in the additional-singleton paradigm (Hickey et al., 2011), we focussed our intertrial analysis on colour. Trials were sorted into a "same" condition, corresponding to trials in which the colour that defined the target was the same as the one that had defined the target in the previous trial (and vice versa), and a "swap" condition, corresponding to trials on which the colour that defined the target was the same as the one that had defined the distractor in the previous trial (and vice versa). As illustrated in Fig. 3, both same-colour and colourswap conditions showed a proximity effect on RTs. The initial statistical analysis took the form of a two-way RANOVA with the factors Proximity and Intertrial Condition (same colour vs. colour swap). Main effects of proximity $[F(4,52)=5.19, p=.001]$ and intertrial condition $[F(1,13)=$ $27.78, p<.001]$ were revealed. Critically, these factors did not interact $[F(4,52)<1]$, and planned follow-up $t$ tests revealed that proximity had a significant effect on RT in each of the "same" $[F(4,52)=4.11, p=.006]$ and "swap" conditions $[F(4,52)=2.639, p=.044]$ when examined in isolation. There were no apparent intertrial patterns in error rates, although this might have been due to a floor effect: Error rates were very low in some combinations of proximity and intertrial condition, reducing variability and making statistical analysis problematic.

In calculating hemispheric effects, there are difficulties in analysis for stimuli located on the vertical meridian of the search display. Stimulus positions on the vertical

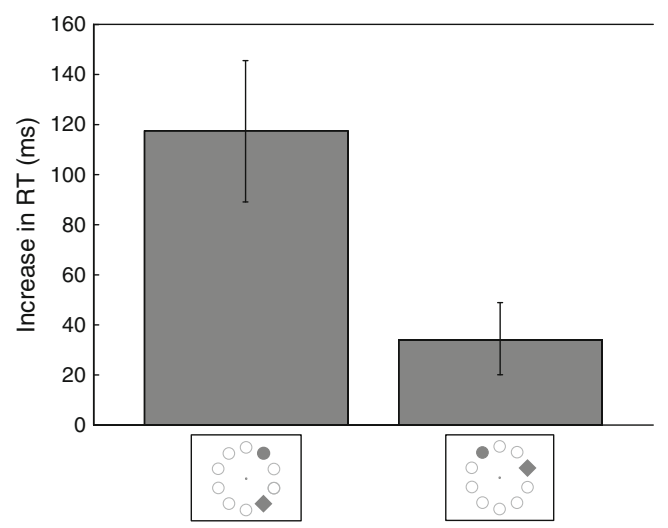

Fig. 3 Results from the overall hemispheric analysis for Experiment 1 meridian are represented in both visual cortices, and as a result it becomes unclear whether a stimulus on the vertical meridian should be considered as in the "same" or in the "opposite" hemisphere. We circumvented this problem by excluding from the analysis of hemispheric effects trials on which the target or distractor was presented on the vertical meridian of the display. In order to further ensure that the hemispheric effect was not confounded with the proximity effect described above, we analysed only trials on which the target and salient distractor were separated by two lowsalience distractors. As illustrated in Figs. 4 and 5, this analysis demonstrated that median RT was significantly reduced when the target and distractor were presented to contralateral hemifields $(789 \mathrm{~ms})$, as compared to when they were presented in the same hemifield $[823 \mathrm{~ms} ; t(13)=$ $2.48, p=.028]$. No effects were identified in the analysis of error rates $[t(13)=1.70, p=.11]$.

Intertrial analysis of hemispheric effects revealed numerically slower RTs in the same-hemisphere condition for both same-colour and colour-swap trials (see Fig. 5). A two-way RANOVA with the factors Intertrial Condition (same colour vs. colour swap) and Hemisphere Condition (same hemifield vs. contralateral hemifields) revealed a main effect of intertrial condition $[F(1,13)=28.40, p<.001]$. Neither the main effect of hemisphere $[F(1,13)=2.63, p=.129]$ nor the interaction $[F(1,13)=1.77, p=.207]$ was significant, but a planned $t$ test comparison of same versus contralateral hemisphere in the swap condition indicated the reliability of this simple effect $[t(13)=2.69, p=.019]$. There were no apparent effects in error rates. As in the intertrial analysis of proximity above, low variability in error rates across conditions made statistical analysis of the error data problematic.

In the experimental design employed in Experiment 1, the target shape could be either a diamond (among circles) or a circle (among diamonds), raising the possibility of intertrial effects tied to repetition of the shapes of the target and distractor (rather than repetition of the target and distractor colour, the results of which are reported above). An RT analysis of the intertrial effects related to target and distractor shape revealed no significant effects [proximity intertrial condition: $F(1,13)<1$; proximity, $F(4,52)=5.37$, $p=.001$; interaction, $F(4,52)<1$; hemisphere intertrial condition: $F(1,13)<1$; hemisphere, $F(1,13)=3.662, p=$ .078 ; interaction, $F(1,13)=1.59, p=.230]$.

\section{Discussion}

We approached experimentation with the idea that there might be two sources of distractor cost in the additionalsingleton paradigm, namely the capture of attention and a static distractor cost, possibly associated with a process of perceptual filtering. Our expectation was that capture would vary as a function of intertrial condition, such that RF- 


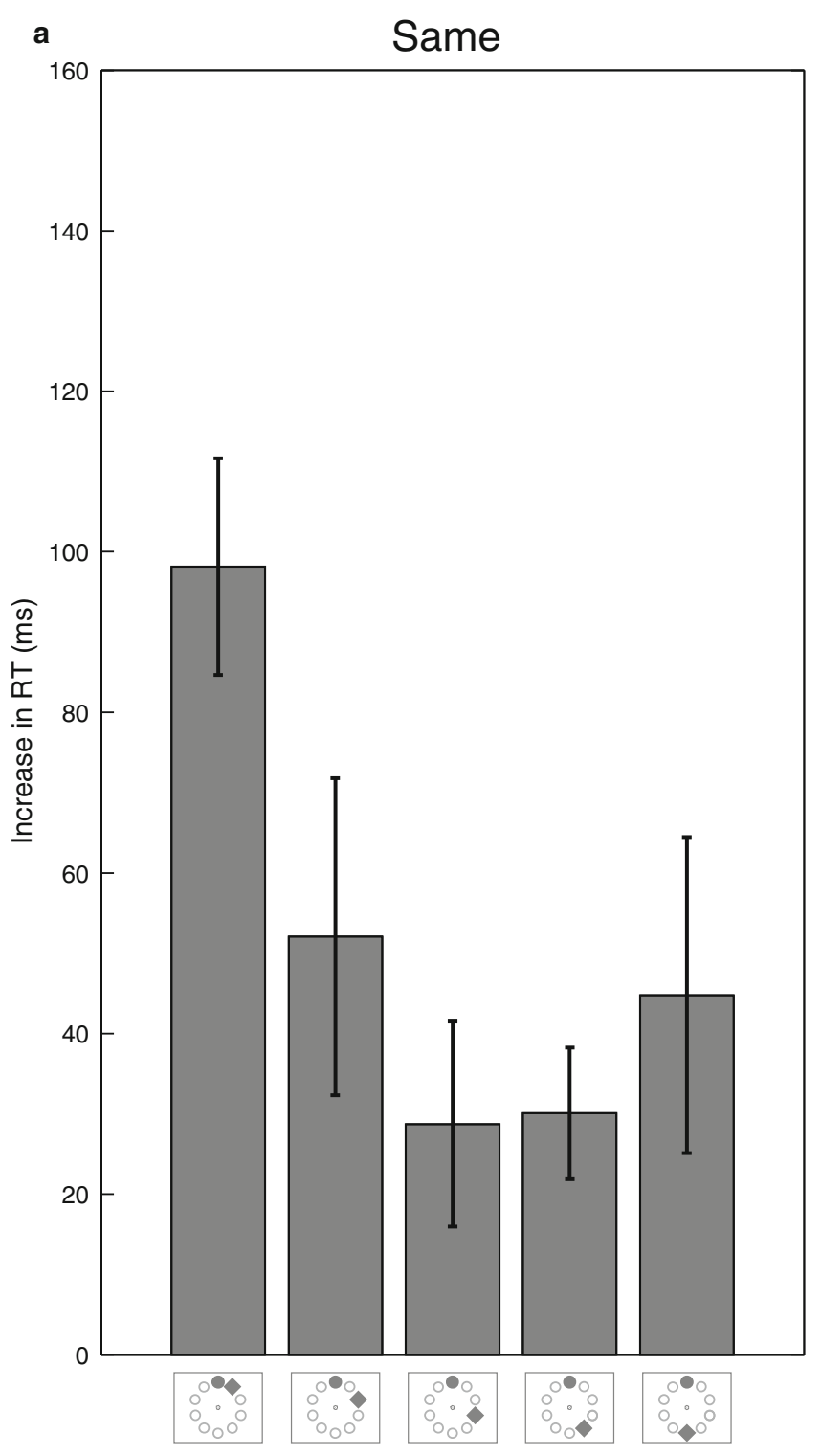

Fig. 4 Intertrial analysis of proximity results for Experiment 1. (a) Results from trials on which the colours defining the target and the distractors were the same as in the immediately preceding trial. (b)

scaled proximity hemispheric effects would increase in the "swap" condition, but that static distractor costs possibly tied to perceptual filtering would remain the same in both "same" and "swap" conditions.

The results of this experiment were exactly the opposite. RF-scaled proximity and hemispheric effects did not differ as a function of intertrial condition, but there was a clear main effect: The static distractor cost identified in the swap-colour condition was reliably larger than that identified in the same-colour condition. It appears that capture occurs in both the same-colour and swap-colour conditions. Differences in behaviour as a function of intertrial contingency appear to reflect something other than capture.

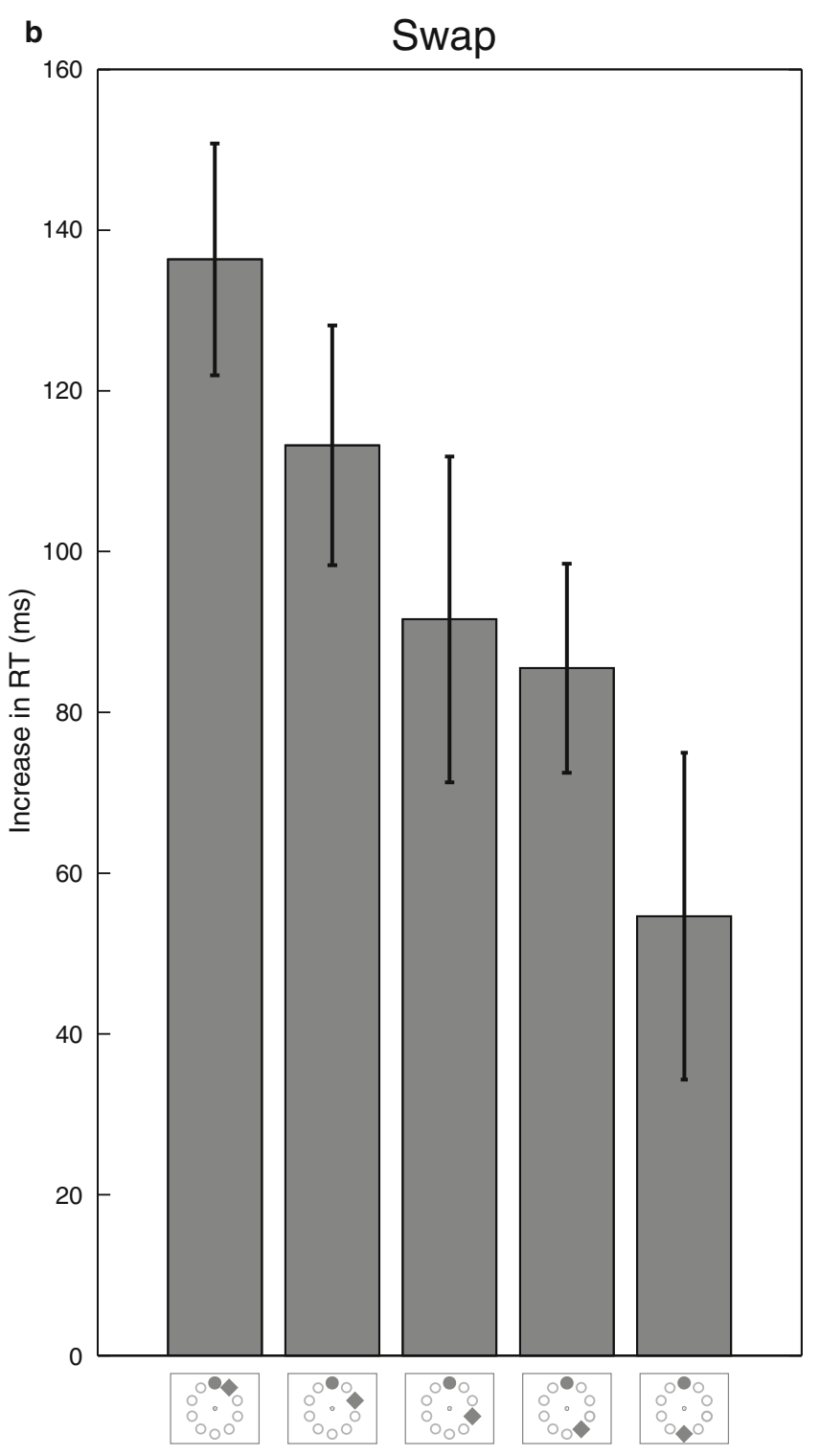

Results from trials on which the colours defining the target and the distractors were swapped relative to the immediately preceding trial

This suggests the need for a reconsideration of the effect of feature priming on capture. However, some limitations to the logic of Experiment 1 should be addressed first. The first of these is that Experiment 1 was grounded on the assumption that RF-scaled distractor interference in the additional-singleton paradigm is necessarily caused by capture. Although this idea is consistent with the existing literature (Mounts, 2000b, 2005; Mounts \& Gavett, 2004) and has been demonstrated under different circumstances by Mounts (2000a), there is still a possibility that perceptual interference plays a role in RF-scaled effects under the specific task confines created by the present experimental paradigm. The second limitation is that Experiment 1 employed mixed blocks. In mixed 


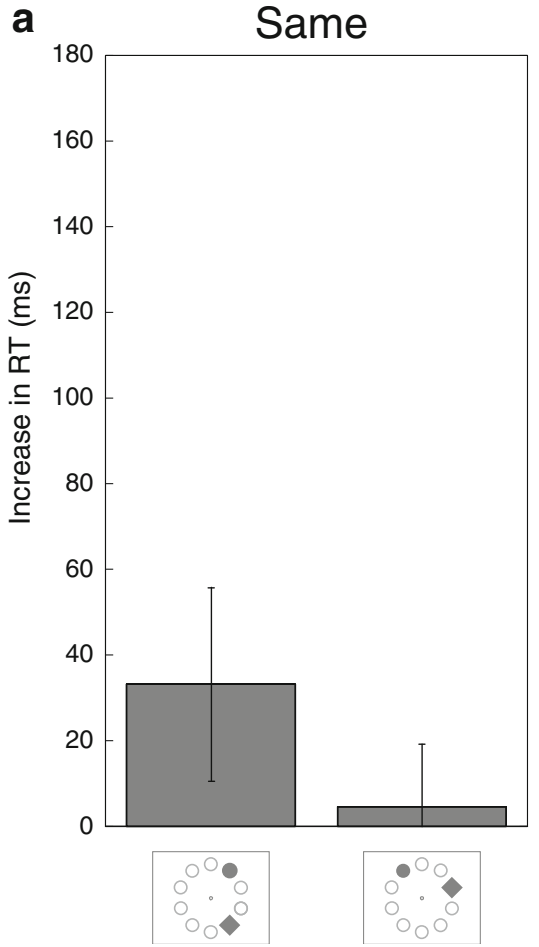

Fig. 5 Intertrial analysis of hemispheric results for Experiment 1. (a) Results from trials on which the colours defining the target and the distractors were the same as in the immediately preceding trial. (b)

blocks, participants are unable to establish an attentional set for target features (and against distractor features) because they cannot be certain which features will define the target in any given trial. Under these circumstances, the attentional system may revert to a set that prioritises salience-something Bacon and Egeth (1994) termed singleton detection mode. If participants were in such a state, evidence of capture in samecolour trials would not convincingly demonstrate that distractor costs observed under similar circumstancessuch as pure blocks - would also reflect the capture of attention.

We addressed these two issues in a second experiment. Experiment 2 differed from Experiment 1 in two critical ways. First, we included a condition in which participants searched for a colour singleton and ignored a shape singleton. In these circumstances, search is for the most salient object in the search display, and capture does not occur (Theeuwes, 1992). If RF-scaled distractor effects are caused by capture, these costs should be absent in the colour-singleton target condition. Second, in Experiment 2 the target was always a circle, distractors were always diamonds, and the colours that defined the target and distractor stayed the same throughout each block. Participants were thus afforded the opportunity to adopt an attentional set specific to target features and against distractor features.

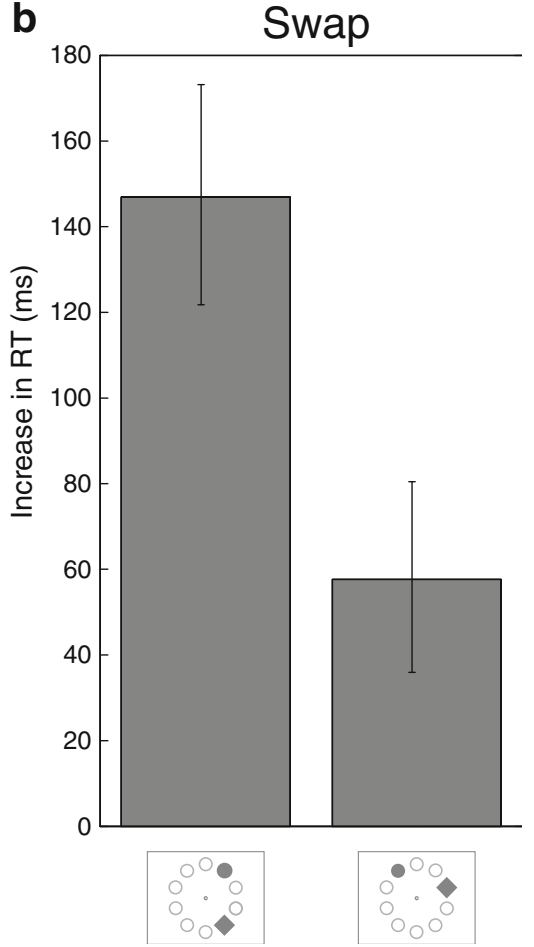

Results from trials on which the colours defining the target and the distractors were swapped relative to the immediately preceding trial

\section{Experiment 2}

\section{Method}

Participants A group of 12 neurologically normal volunteers were financially compensated for participation. One participant was excluded because of excessively long RTs $(>2,500 \mathrm{~ms}$ median RT), and another because of an apparent inability to complete the task while maintaining eye fixation. Of the remaining 10 participants, 9 (5 women, 4 men; mean age $=20$ years $\pm 2.8 S D$ ) were right-handed.

Stimuli and procedure In Experiment 2, both the shape singleton and the colour singleton were present in every trial. The shape singleton was in all cases a circle (with all distractors diamonds), and the colour singleton was defined by the same colour throughout each block of 48 trials. Participants were informed of the target colour at the beginning of each block of trials and saw equal numbers of blocks for the two possible colour mappings (red targetgreen distractor and vice versa).

The shape singleton was defined as the target for only half of the experiment; in the remaining trials, response was based on the line contained within the colour singleton. All participants completed 15 blocks of 48 trials in the shapetarget condition prior to completing the same number of blocks and trials in the colour-target condition. Participants 
were informed of the change in task requirements only at the halfway point in the experiment (minimising the possibility that the colour singleton would be viewed by participants as task relevant in the first half of the experiment). As in Experiment 1, an EOG was recorded during participation, and $9 \%$ of correct-response trials were rejected due to eye movements. Another $1 \%$ of trials were discarded due to response latencies exceeding $1.35 \mathrm{~s}$. All other parameters were as in Experiment 1.

Results

Proximity analysis was conducted on RT data for each of the shape-target and colour-target conditions, with trials further separated into same-hemifield and oppositehemifield conditions. Results from this analysis are illustrated in Fig. 6. Visual examination suggests the presence of at least two effects: a main effect of target type, with participants slower to respond to the shape target across all conditions, and an effect of proximity in the shape-target, same-hemifield condition, with RTs increasing as target and salient distractor became closer. A RANOVA with the factors Target Type (colour vs. shape singleton), Shared Hemifield (same hemifield vs. contralateral hemifields), and Proximity (five levels) was conducted to statistically assess the data. Three significant effects were identified: a main effect of target type $[F(1,9)=20.95, p<.001]$, reflecting slower responses in the shape-target condition; an interaction between the Target Type and Proximity factors

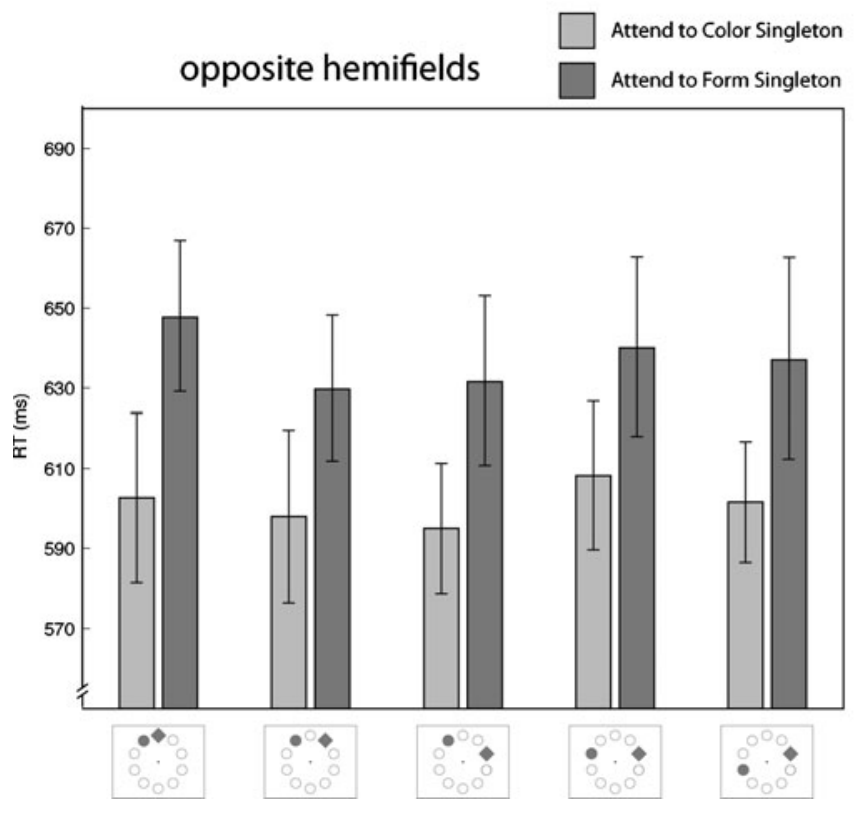

Fig. 6 Results from a hemispheric analysis for Experiment 2. (a) Results from trials on which the target and the singleton distractor were presented to contralateral visual hemifields. (b) Results from
$[F(4,36)=17.62, p<.001]$, reflecting an increase in RTs as a function of increasing proximity in the shape-target condition; and a marginally significant interaction between all three factors $[F(4,36)=2.49, p=.060]$, reflecting an increase in the proximity effect in the shape-target, samehemifield condition relative to all other conditions.

In this three-way ANOVA, no significant effect of shared hemifield was identified. However, there is a problem with this analysis: It included trials on which stimuli were presented on the vertical meridian of the display. As outlined in the Results section of Experiment 1, stimuli presented on the vertical meridian are not optimal for hemispheric analyses because they are represented in both visual cortices. With this in mind, we conducted an additional analysis. This was identical to the hemispheric analysis conducted in Experiment 1; only trials on which neither stimulus was on the vertical meridian and the two salient objects were separated by two stimulus positions were included. As illustrated in Fig. 7, the results suggest that participants were slower to respond to the target when it was presented in the same hemifield as the distractor, both when they searched for the shape singleton and when they searched for the colour singleton. A two-way RANOVA revealed a main effect of target type $[F(1,9)=$ $7.08, p=.026]$, reflecting an increase in RTs in the shapetarget condition, and a main effect of hemifield $[F(1,9)=$ $9.29, p=.014]$, reflecting an increase in RTs in the samehemifield condition, but no interaction between these factors $(F<1)$. No patterns were identified in error rates, which were generally low across all conditions.

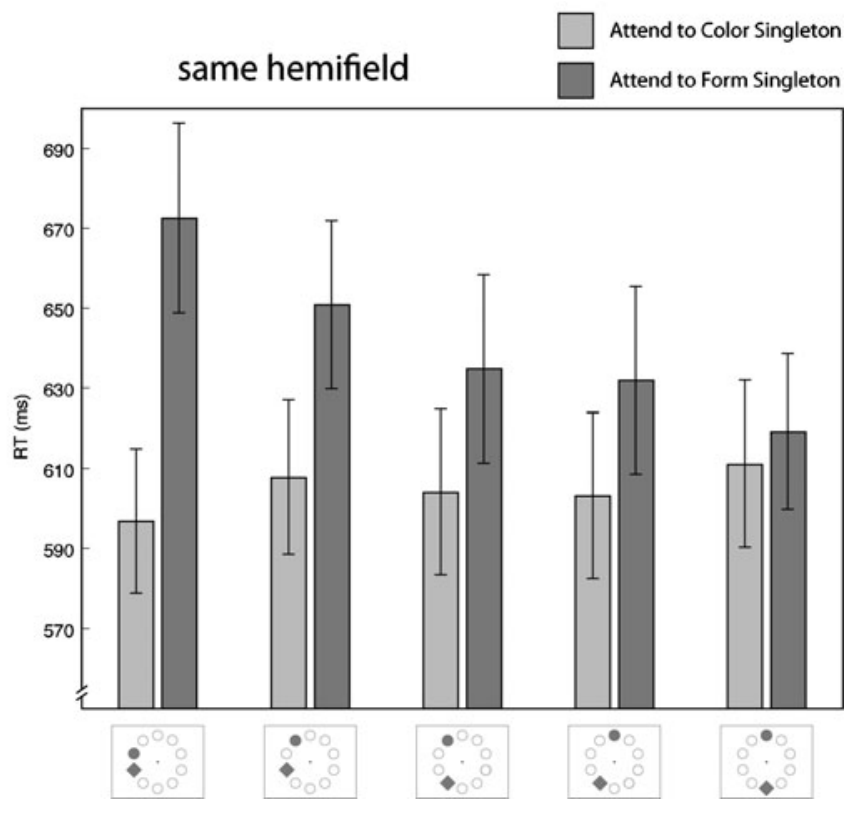

trials on which the target and the singleton distractor were presented to the same visual hemifield 


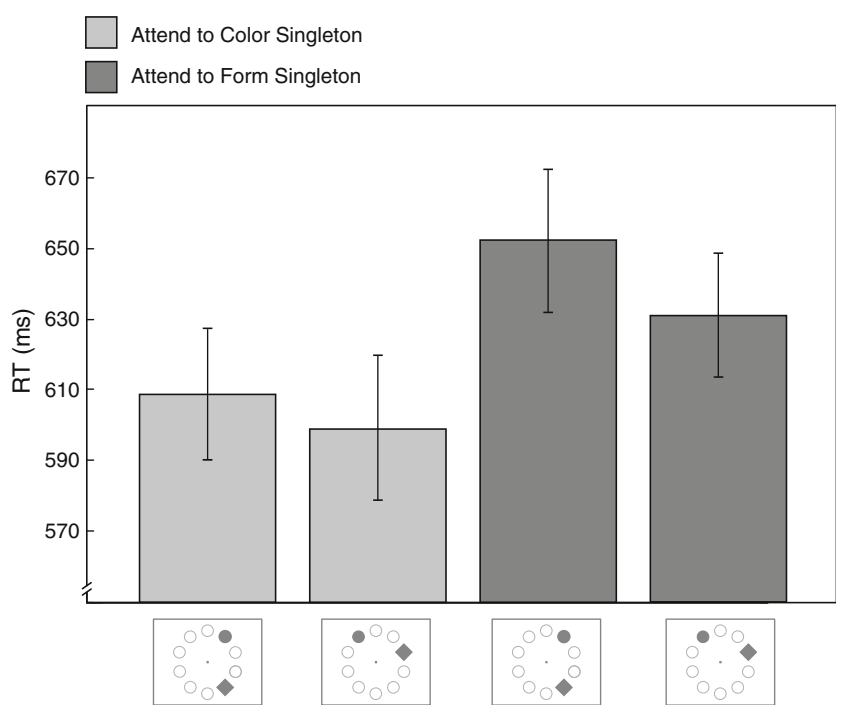

Fig. 7 Results from the overall hemispheric analysis for Experiment 2

\section{Discussion}

Experiment 2 garnered three relevant findings. First, RFscaled proximity distractor costs were observed only in the shape-singleton target condition, and hemispheric effects were numerically larger in this condition. Prior work had demonstrated that a shape singleton does not capture attention when search is for a colour singleton (Theeuwes, 1991), and these results are consistent with the idea that that RF-scaled effects (or at least proximity effects) constitute an index of capture (as established in previous studies - e.g., Caputo \& Guerra, 1998; Mounts, 2000a, 2000b, 2005; Mounts \& Gavett, 2004; McCarley \& Mounts, 2007, 2008; McCarley et al., 2007). ${ }^{2}$

A caveat must be attached to this proposal: Though Experiment 2 provided a clear relationship between proximity effects and capture, the relationship of hemispheric effects to capture is more ambiguous. Hemispheric effects were numerically reduced in the colour-target condition of Experiment 2 (see Fig. 7), but this was not significant. Close examination of the residual effect in the "attend to colour singleton" condition suggests that this effect can be traced to the behaviour of 3 participants. In comparison, the hemispheric effect in the "attend to form singleton" condition was evident in all participants. One

\footnotetext{
${ }^{2}$ That is to say, when these effects are quite large, as in the present study, they likely index capture. There very likely are also proximity costs associated with competitive suppression that occur when capture does not occur (see Bahcall \& Kowler, 1999; Cave \& Zimmerman, 1997). However, these effects are at least an order of magnitude smaller than those created by capture (e.g., $<10 \mathrm{~ms}$ in Cave \& Zimmerman, 1997).
}

possibility is these 3 participants attended to the shape distractor in some subset of trials during search for the colour target. Fukuda and Vogel (2009) recently demonstrated that there are substantial individual differences in the degrees to which salient irrelevant singletons affect search, though little research has been conducted examining variability in the impact of a distractor that is less salient than the target. The present results raise the possibility that some participants may be particularly sensitive to interference from these items.

In any case, proximity costs appear a reliable index of capture, even if the relationship between hemispheric effects and capture is less clear. The second central finding of Experiment 2 was that this index of capture was evident in a blocked version of the additional-singleton paradigm in which the target and distractor features did not vary from trial to trial. This suggests that attention is captured by the salient distractor in this version of the task, consistent with results from the "same" intertrial condition of Experiment 1.

Finally, capture-related proximity costs were evident in the shape-singleton target condition of Experiment 2, even though the features that defined the target and the salient distractor in this blocked version of the additional-singleton paradigm were predictable, and participants were therefore able to establish a feature-based attentional set (e.g., feature search mode; Bacon \& Egeth, 1994). This means that the presence of RF-scaled effects in Experiment 1 was not a byproduct of the experimental design; participants might have been forced to adopt a salience-based search strategy in Experiment 1, and this may have been responsible for the capture observed in same-colour trials, but the results of Experiment 2 demonstrate RF-scaled effects when a saliencebased search strategy was neither necessary nor beneficial.

\section{General discussion}

In pure blocks of the additional-singleton paradigm, when the features that define a target and salient distractor do not vary from trial to trial, the behavioural costs associated with the presence of the salient distractor are relatively small (Bacon \& Egeth, 1994; Theeuwes, 1992). In mixed blocks, when features that previously characterised a distractor can define a target (and vice versa), distractor costs are relatively large (Hickey et al., 2006; Theeuwes, 1991). Recent intertrial studies of visual search have suggested that this difference stems from trials in mixed blocks on which the features that define the target and distractor swap between trials. Responses on these trials are slow and error prone. In contrast, responses on "same" trials are similar to those observed in pure blocks (Hickey et al., 2011; Pinto et al., 2005). This suggests that capture occurs in trials on 
which the perceptual features that characterise the distractor have recently characterised the target. Based on this idea, we approached the present experiments with the expectation that RF-scaled interference effects-which index attentional capture - would be evident only under these circumstances. We further expected that the main effects of distractor presence - costs that do not scale as a function of shared RF, and that might reflect processes of perceptual filtering and resolution triggered by the distractor-would not vary as a function of temporal context.

The results were exactly opposite to our expectations. The idea that RF-scaled interference would be evident only when target and distractor colours swapped predicted interactions between the factor Intertrial Condition (same vs. swap) and measures of RF-scaled interference (proximity or shared visual hemifield), but the results showed no evidence of this interaction: RF-scaled interference was of similar magnitudes in both same-colour and colour-swap trials. On the other hand, the idea that non-RF-scaled costs would stay the same in the two conditions predicted the absence of a main effect of intertrial condition on the cost of distractor presence, but the results showed a clear main effect: The distractor interfered with search to a greater degree in the swap trials.

Experiment 2 showed that RF-scaled costs were evident in a blocked version of the additional-singleton paradigm in which the features defining the target and distractor did not vary from trial to trial. These are circumstances in which participants might be able to adopt a feature-based search strategy, and these results are therefore consistent with the idea that participants use salience to guide search even when better strategies are available to them (cf. Bacon \& Egeth, 1994).

The presence of RF-scaled distractor costs under circumstances in which we did not expect capture to occur might suggest that these costs are inadequate indices of capture, but in Experiment 2 we found that these costs were reduced or eliminated when capture did not occur. ${ }^{3}$ This is consistent with other results in the literature (e.g., Mounts, 2000a).

\footnotetext{
${ }^{3}$ During the editorial process, an anonymous reviewer suggested that the absence of attentional capture in the colour-singleton-target condition of Experiment 2 might reflect training effects in the visual search task. Prior research has shown that participants can gain the ability to ignore task-irrelevant distractor stimuli with increased task experience (Kim \& Cave, 1999). In Experiment 2, the coloursingleton-target condition followed the shape-singleton-target condition for all participants, who were thus more familiar with the search task in this condition; practice could have played a role in reducing distractor interference. However, this does not directly impact our conclusions. We claim that the absence of capture in the colour-target condition is reflected in the absence of proximity effects; this continues to be the case, regardless of whether the absence of capture reflects practice or the fact that the target in this condition was more salient than the distractor.
}

Attentional capture thus appears to be more robust and pervasive than we had assumed in approaching this study, or than has been suggested by our earlier studies of intertrial contingency (i.e., Hickey et al., 2011; Pinto et al., 2005). For example, in Hickey et al. (2011), we showed that an event-related potential (ERP) index of attentional selection - the N2pc-is elicited by distractor stimuli in "swap" trials but not in "same" trials. We interpreted this as evidence that attentional capture occurs largely under circumstances in which the distractor is defined by features that have recently characterised the target. Our logic was that the absence of an N2pc indicated the absence of attention.

The present results motivate an alternative interpretation, namely that the difference in the distractor-elicited N2pc we observed in our earlier study - presence in "swap" trials and absence in "same" trials - may reflect a qualitative difference in the attentional mechanisms activated by the distractor, rather than a quantitative difference in the degree of capture. According to this idea, the type of attentional mechanism activated by a "swap" distractor might be reflected in an N2pc, whereas the type of attentional mechanism activated by a "same" distractor might not be clearly indexed in the ERP.

This possibility is lent some support by our observation of a main effect of intertrial contingency in Experiment 1 of the present study; if "extra" attentional mechanisms were activated in response to a "swap" distractor, which meant that attention dwelled on the distractor longer, but this dwell had no impact on competitive target-distractor interactions, this would create a main effect of intertrial contingency without an interaction, as is observed in the present results. However, this is clearly conjecture, and dedicated ERP work will be needed to determine the relationship between the $\mathrm{N} 2 \mathrm{pc}$ and RF-scaled interference (see Hillimire, Mounts, Parks, \& Corballis, 2009, for an initial study of this relationship).

In Experiment 2, proximity effects were limited to conditions in which the target and distractor were presented to the same visual hemifield. Similar results have been reported by Mounts and Gavett (2004), though under very different experimental circumstances and with accuracy, rather than RT, as a dependent measure. This general finding is in line with results from studies of primate electrophysiology. For example, Chelazzi, Duncan, Miller, and Desimone (1998) examined neural competition in macaque inferior temporal cortex. These authors found that the neural response to an effective stimulus was reduced by the presentation of a competing stimulus only when both objects were presented to one hemifield. Moreover, the neural response to an effective distractor was inhibited only when attention was deployed in the same hemifield. Results from Chelazzi et al. (1998), alongside the present findings 
and those of Mounts and Gavett (2004), suggest that stimuli presented to contralateral hemifields interfere with one another to a surprisingly small degree.

In the introduction, we raised the idea that the capture of attention may reflect the outcome of competitive processing in visual cortex that occurs prior to the establishment of endogenous biases. In line with this, recent studies examining the time course of covert and overt selection have suggested that capture occurs when selection is initiated quickly. For example, short-latency saccadic eye movements that are started soon after stimulus presentation are deployed to the location of a salient, task-irrelevant object before being redirected to the target. Longer latency movements go directly to the target (van Zoest, Donk, \& Theeuwes, 2004). Similar results have been observed in manual responses (Hunt, von Mühlenen, \& Kingstone, 2007), and human electrophysiological work has tied this pattern to the underlying deployment of covert attention (Hickey, van Zoest, \& Theeuwes, 2010). Results from the animal literature show that endogenous attentional set for a specific colour does not affect visual response in monkey V4 neurons until after the onset of the exogenous response (Chelazzi, Duncan, Miller, \& Desimone, 1998; Reynolds \& Desimone, 2003).

Time thus appears to play an important role in the integration of exogenous and endogenous biases in visual processing. Our operating hypothesis is that endogenous control relies on feedback connections from higher-level visual cortex on lower-level areas. Reentrant processing of this nature might rely on the afferent, feed-forward sweep of information through visual cortex in order to be initiated, thus creating a brief delay between the beginning of visual processing and the establishment of top-down set (and as suggested in the model of visual attention proposed by Tsotsos et al., 1995). Though this delay may be very short, any processes initiated during this time would necessarily be based on stimulus characteristics rather than on prior knowledge.

In conclusion, the present study demonstrates that attention is captured by a salient colour singleton even under circumstances in which the characteristics defining this object do not vary from trial to trial, and this can be indexed in RF-scaled interference costs.

Open Access This article is distributed under the terms of the Creative Commons Attribution Noncommercial License which permits any noncommercial use, distribution, and reproduction in any medium, provided the original author(s) and source are credited.

\section{References}

Bacon, W. F., \& Egeth, H. E. (1994). Overriding stimulus-driven attentional capture. Perception \& Psychophysics, 55, 485-496.
Bahcall, D. O., \& Kowler, E. (1999). Attentional interference at small spatial separations. Vision Research, 39, 71-86. doi:10.1016/ S0042-6989(98)00090-X

Beck, D. M., \& Kastner, S. (2005). Stimulus context modulates competition in human extrastriate cortex. Nature Neuroscience, 8, 1110-1116.

Broadbent, D. E. (1958). Perception and communication. New York: Oxford University Press.

Burnham, B. R. (2007). Displaywide visual features associated with a search display's appearance can mediate attentional capture. Psychonomic Bulletin \& Review, 14, 392-422.

Caputo, G., \& Guerra, S. (1998). Attentional selection by distractor suppression. Vision Research, 38, 669-689.

Cave, K. R., \& Zimmerman, J. M. (1997). Flexibility in spatial attention before and after practice. Psychological Science, 8, 399-403. doi:10.1111/j.1467-9280.1997.tb00433.x

Chelazzi, L., Duncan, J., Miller, E. K., \& Desimone, R. (1998). Responses of neurons in inferior temporal cortex during memoryguided visual search. Journal of Neurophysiology, 80, 2918 2940.

Chelazzi, L., Miller, E. K., Duncan, J., \& Desimone, R. (1993). A neural basis for visual search in inferior temporal cortex. Nature, $363,345-347$.

de Fockert, J., Rees, G., Frith, C., \& Lavie, N. (2004). Neural correlates of attentional capture in visual search. Journal of Cognitive Neuroscience, 16, 751-759. doi:10.1162/089892904970762

Desimone, R., \& Duncan, J. (1995). Neural mechanisms of selective visual attention. Annual Review of Neuroscience, 18, 193-222.

Desimone, R., \& Ungerleider, L. G. (1989). Neural mechanisms of visual processing in monkeys. In F. Boller \& J. Grafman (Eds.), Handbook of neuropsychology, vol. 2 (pp. 267-299). Amsterdam: Elsevier.

Folk, C. L., \& Remington, R. (1998). Selectivity in distraction by irrelevant featural singletons: Evidence for two forms of attentional capture. Journal of Experimental Psychology. Human Perception and Performance, 24, 847-858.

Fukuda, K., \& Vogel, E. K. (2009). Human variation in overriding attentional capture. Journal of Neuroscience, 29, 8726-8733. doi:10.1523/JNEUROSCI.2145-09.2009

Hickey, C., Chelazzi, L., \& Theeuwes, J. (2010). Reward changes salience in human vision via the anterior cingulate. Journal of Neuroscience, 30, 11096-11103.

Hickey, C., McDonald, J. J., \& Theeuwes, J. (2006). Electrophysiological evidence of the capture of visual attention. Journal of Cognitive Neuroscience, 18, 604-613.

Hickey, C., Olivers, C. N. L., Meeter, M., \& Theeuwes, J. (2011). Feature priming and the capture of visual attention: Linking two ambiguity resolution hypotheses. Brain Research, 1370, 175184. doi:10.1016/j.brainres.2010.11.025

Hickey, C., van Zoest, W., \& Theeuwes, J. (2010). The time course of exogenous and endogenous control of covert attention. Experimental Brain Research, 201, 789-796.

Hillimire, M. R., Mounts, J. R., Parks, N. A., \& Corballis, P. M. (2009). Competitive integration degrades target selection: An ERP study. Psychophysiology, 46, 1080-1089.

Hillstrom, A. P. (2000). Repetition effects in visual search. Perception \& Psychophysics, 62, 800.

Hunt, A. R., von Mühlenen, A., \& Kingstone, A. (2007). The time course of attentional and oculomotor capture reveals a common cause. Journal of Experimental Psychology. Human Perception and Performance, 33, 271-284. doi:10.1037/00961523.33.2.271

Kahneman, D., Treisman, A., \& Burkell, J. (1983). The cost of visual filtering. Journal of Experimental Psychology. Human Perception and Performance, 9, 510-522. doi:10.1037/00961523.9.4.510 
Kastner, S., De Weerd, P., Desimone, R., \& Ungerleider, L. G. (1998). Mechanisms of directed attention in the human extrastriate cortex as revealed by functional MRI. Science, 282, 108-111.

Kastner, S., De Weerd, P., Pinsk, M. A., Elizondo, M. I., Desimone, R., \& Ungerleider, L. G. (2001). Modulation of sensory suppression: Implications for receptive field sizes in the human visual cortex. Journal of Neurophysiology, 86, 1398-1411.

Kim, M. S., \& Cave, K. R. (1999). Top-down and bottom-up attentional control: On the nature of interference from a salient distractor. Perception \& Psychophysics, 61, 1009-1023.

Luck, S. J., Girelli, M., McDermott, M. T., \& Ford, M. A. (1997). Bridging the gap between monkey neurophysiology and human perception: An ambiguity resolution theory of visual selective attention. Cognitive Psychology, 33, 64-87.

Maljkovic, V., \& Nakayama, K. (1994). Priming of pop-out: I. Role of features. Memory \& Cognition, 22, 657-672.

McCarley, J. S., \& Mounts, J. R. W. (2007). Spatially mediated capacity limits in attentive visual perception. Acta Psychologica, 126, 98-119.

McCarley, J. S., \& Mounts, J. R. W. (2008). On the relationship between flanker interference and localized attentional interference. Perception, 128, 102-109.

McCarley, J. S., Mounts, J. R. W., \& Kramer, A. F. (2007). Spatially mediated capacity limits in attentive visual perception. Acta Psychologica, 126, 98-119. doi:10.1016/j.actpsy.2006.11.004

Miller, E. K., Gochin, P. M., \& Gross, C. G. (1993). Suppression of visual responses of neurons in inferior temporal cortex of the awake macaque by addition of a second stimulus. Brain Research, 616, 25-29.

Moran, J., \& Desimone, R. (1985). Selective attention gates visual processing in the extrastriate cortex. Science, 229, 782-784.

Mounts, J. R. W. (2000a). Attentional capture by abrupt onsets and feature singletons produces inhibitory surrounds. Perception \& Psychophysics, 62, 1485-1493. doi:10.3758/BF03212148

Mounts, J. R. W. (2000b). Evidence for suppressive mechanisms in attentional selection: Feature singletons produce inhibitory surrounds. Perception \& Psychophysics, 62, 969-983. doi:10.3758/BF03212082
Mounts, J. R. W. (2005). Attentional selection: A salience-based competition for representation. Perception \& Psychophysics, 67, 1190-1198. doi:10.3758/BF03193552

Mounts, J. R. W., \& Gavett, B. E. (2004). The effects of salience on localized attentional inhibition. Vision Research, 44, 1575-1588.

Pinto, Y., Olivers, C. N. L., \& Theeuwes, J. (2005). Target uncertainty does not lead to more distraction by singletons: Intertrial priming does. Perception \& Psychophysics, 67, 1354-1361. doi:10.3758/ BF03193640

Rauschenberger, R. (2003). Attentional capture by auto- and allo-cues. Psychonomic Bulletin \& Review, 10, 814-842.

Reynolds, J. H., Chelazzi, L., \& Desimone, R. (1999). Competitive mechanisms subserve attention in macaque areas V2 and V4. Journal of Neuroscience, 19, 1736-1753.

Reynolds, J. H., \& Desimone, R. (2003). Interacting roles of attention and visual salience in V4. Neuron, 37, 853-863.

Theeuwes, J. (1991). Cross-dimensional perceptual selectivity. Perception \& Psychophysics, 50, 184-193.

Theeuwes, J. (1992). Perceptual selectivity for color and form. Perception \& Psychophysics, 51, 599-606.

Theeuwes, J. (2004). Top-down search strategies cannot override attentional capture. Psychonomic Bulletin \& Review, 11, 65-70.

Theeuwes, J. (2010). Top-down and bottom-up control of visual selection. Acta Psychologica, 135, 77-99. doi:10.1016/j. actpsy.2010.07.006

Torralbo, A., \& Beck, D. M. (2008). Perceptual load-induced selection as a result of local competitive interactions in visual cortex. Psychological Science, 19, 1045-1050.

Treisman, A. M. (1969). Strategies and models of selective attention. Psychological Review, 76, 282-299. doi:10.1037/h0027242

Tsotsos, J. K., Culhane, S. M., Kei Wai, W. Y., Lai, Y., Davis, N., \& Nuflo, F. (1995). Modeling visual attention via selective tuning. Artificial Intelligence, 78, 507-545.

van Zoest, W., Donk, M., \& Theeuwes, J. (2004). The role of stimulus-driven and goal-driven control in saccadic visual selection. Journal of Experimental Psychology. Human Perception and Performance, 30, 746-759. doi:10.1037/00961523.30.4.749 